\title{
Application of Sentinel-1 radar remote sensing data for detecting nuclear test location in North Korea on September 3, 2017
}

\author{
Akhmadiya Asset ${ }^{a}$, Moldamurat Khuralay $^{b}{ }$, Nabiyev Nabi ${ }^{a}{ }$, Kismanova Aigerim ${ }^{a}$ \\ ${ }^{a}$ Kazakh Agrotechnical University, Nur-Sultan, Kazakhstan \\ ${ }^{b}$ Eurasian National University, Nur-Sultan, Kazakhstan \\ E-mail:a.akhmadiya@kazatu.kz
}

\begin{abstract}
Sentinel-1A/B radar remote sensing data were applied for the first time to determine the sixth nuclear test, its underground explosion h-bomb location and affected zone in North Korea, on September 3, 2017. Location of epicenters nuclear test were found according to line-of-sight displacement images via its maximum value. Line-of-sight displacement images were obtained by processing in the GMTSAR package in the VirtualBox virtual machine of the Linux Ubuntu 16.04 operation system. In this research, three scenes Sentinel-B data with descending orbits were considered, one after and two before the event (the nuclear test date) scene were used.
\end{abstract}

Keywords: Sentinel-1, radar image processing, line-of-sight displacement, nuclear test, North Korea.

\section{Introduction}

North Korea claimed to have tested an h-bomb for the first time, with an underground explosion on September 3, 2017. Seismic sensors of neighboring countries registered an earthquake with a magnitude of $6.3 \mathrm{M}$; however, this was not the consequence of an earthquake [1,2]. After that event, seismologists began to study the explosion of an h-bomb in-depth, epicenter, and the power of the explosion in TNT equivalent [3, 4]. Radar Remote Sensing ALOS-2 and TerraSAR-X data were used to determine the epicenter's location [5-8]. Earthquake or underground explosions h-bomb can cause deformation of the earth surface, and this deformation can be found via radar remote sensing data $[9,10]$. ALOS-2 and TerraSAR-X data are not freely available for end-users than Sentinel-1 radar remote sensing data proposed by European Space Agency. Interferograms from Sentinel-1A/B data can be generated to form line-of-sight displacement images further. This value's maximum displacement shows the earth's surface deformation related to underground explosions h-bomb, its epicenter. Sentinel-1 data is more applicable for finding that epicenter because satellite scenes have large territory covering, and the maximum repeat pass period is 6-12 days. Sentinel-1 data is excellent for assessing damage from natural and human-made disasters, such as earthquakes and underground nuclear test explosions [11-15]. Radar remote sensing data can be processed by special software, such as GAMMA, ENVI SARscape, ROI_PAC, GMTSAR, and ESA SNAP to find epicenters [16-19]. Some commercial software was licensed, has for more than $\$ 10,000$, and may not be available to the end-user. In contrast, the GMTSAR package, a set of additional GMT scripts developed by David Sandwell and Xiaohua Xu of the University of California, San Diego, can also be successfully used to determine the epicenter of underground explosion h-bomb. In the future, we will use it to determine the location of the epicenter and show an algorithm for processing remote sensing radar data.

\section{Study area and data}

Three descending orbit scenes of Sentinel-1B before and after the nuclear test event (see Fig. 1) were exploited for finding the epicenter location. Sentinel-1B radar remote sensing satellite operate at a wavelength of approximately $5.6 \mathrm{~cm}$. Radar remote sensing data was downloaded from the EarthData NASA (https://urs.earthdata.nasa.gov/), see Table 1. VV polarization part of Sentinel-1B in Interferometric Wide (IW) swath mode was used to create phase difference with a subtraction of topographic component, absolute unwrapped phase, and line-of-sight images.

Precise orbital coordinates of the satellite's flight and digital elevation model (DEM) is necessary to work with Sentinel-1B data in the GMTSAR package. Updated orbit-file data is available in 20 days for Sentinel-1B satellite data via the website https://qc.sentinel1.eo.esa.int/aux_poeorb/ (see Table 2). Digital elevation model DEM-file can be obtained via the website https://topex.ucsd.edu/gmtsar/demgen/ for the GMTSAR package. 
Table 1. Satellite data

\begin{tabular}{|c|c|c|c|c|}
\hline № & Study area & Date & Orbits & Satellite data \\
\hline 1 & \multirow{3}{*}{$\begin{array}{l}\text { North Korea, } \\
\text { Hamgyong } \\
\text { province, near } \\
\text { Kilju city }\end{array}$} & 17.08.2017 & \multirow{3}{*}{ Descending } & $\begin{array}{l}\text { S1B_IW_SLC_1SDV_20170817T213025_20170817T213052_006985 } \\
\text { _00C4D8_AF51 }\end{array}$ \\
\hline 2 & & 29.08 .2017 & & $\begin{array}{l}\text { S1B_IW_SLC__1SDV_20170829T213026_20170829T213053_007160 } \\
\text { 00C9E7 617F }\end{array}$ \\
\hline 3 & & 10.09 .2017 & & $\begin{array}{l}\text { S1B_IW_SLC__1SDV_20170910T213026_20170910T213053_007335 } \\
\text { 00CF07_6DE9 }\end{array}$ \\
\hline
\end{tabular}

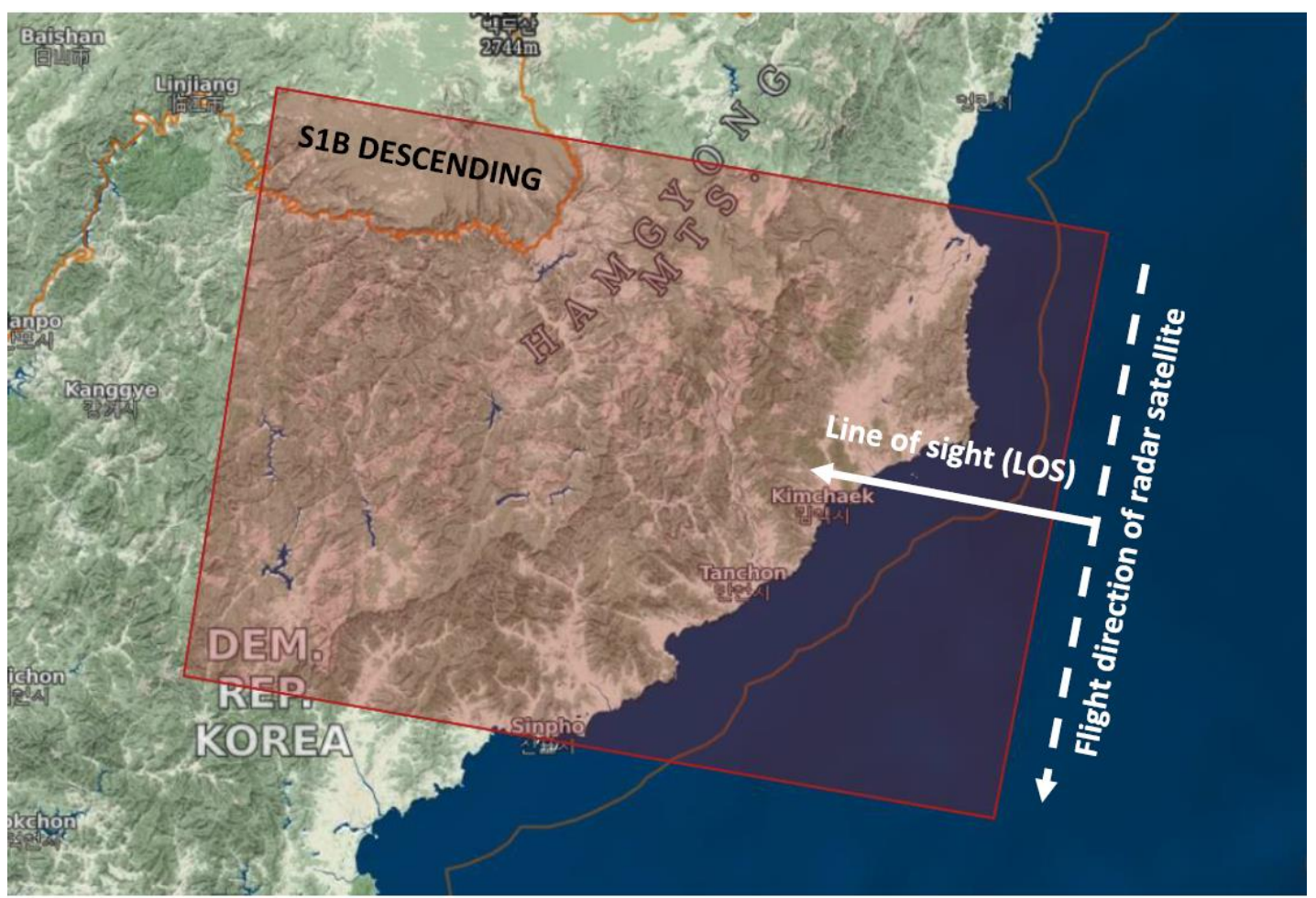

Figure 1. The footprint of selected Sentinel-1B scenes for descending orbits. North Korea, Hamgyong province, near Kilju city

Таблица 2. Satellite data and related orbital files for Sentinel-1B

\begin{tabular}{|c|l|l|}
\hline № & \multicolumn{1}{|c|}{ Satellite data } & \multicolumn{1}{|c|}{ Precise Orbit Ephemerides } \\
\hline 1 & S1B_IW_SLC_1SDV_20170817T213025_20170817T213 & S1B_OPER_AUX_POEORB_OPOD_20170906T11132 \\
& 052_006985_00C4D8_AF51 & 5_V20170816T225942_20170818T005942.EOF \\
\hline 2 & S1B_IW_SLC_1SDV_20170829T213026_20170829T213 & S1B_OPER_AUX_POEORB_OPOD_20170918T11134 \\
& 053_007160_00C9E7_617F & 7_V20170828T225942_20170830T005942.EOF \\
\hline 3 & S1B_IW_SLC_1SDV_20170910T213026_20170910T213 & S1B_OPER_AUX_POEORB_OPOD_20170930T11141 \\
& 053_007335_00CF07_6DE9 & 9_V20170909T225942_20170911T005942.EOF \\
\hline
\end{tabular}

Tree interferometric pairs of Sentinel-1 data were obtained using the GMTSAR package:

1) The first pair for dates: August 29 and September 10, 2017.

2) The second pair for dates: August 17 and September 10, 2017.

\section{Radar image processing}

Radar images are processed using the GMTSAR package. Radar image processing involves the sequential execution of scripts in Fortran and $\mathrm{C}$, one of the most important is the procedure of joint registration of each sub-swath IW1, IW2, IW3, and the formation of an interferogram from Sentinel-1 data [13]. The output products of interferogram formation are the phase difference (see Fig. 2). The phase difference was calculated as follows for each pixel for a pair of jointly co-registered radar images:

$$
\Delta \varphi=\arctan \left(\frac{b_{1}}{a_{1}}\right)-\arctan \left(\frac{b_{2}}{a_{2}}\right)=\varphi_{1}-\varphi_{2}
$$

where $a_{1}, a_{2}-$ the real part of a complex number from a pair of radar images, and $b_{1}, b_{2}$ The imaginary part of each pixel's complex number of these radar images [14]. 
The phase difference is the sum of several components associated with the displacement $\Delta \varphi_{\text {defo }}$, related by topographic phase $\Delta \varphi_{\text {topo }}$, by the atmospheric phase delay $\Delta \varphi_{\text {atmo }}$ and noise effect $\Delta \varphi_{\text {noise }}$ [15]:

$$
\Delta \varphi=\Delta \varphi_{\text {defo }}+\Delta \varphi_{\text {topo }}+\Delta \varphi_{\text {atmo }}+\Delta \varphi_{\text {noise }}
$$

Underground explosion h-bomb can cause a displacement of the Earth's surface, where the phase associated with the displacement can be larger than the phase associated with the atmosphere and noise $\Delta \varphi_{\text {defo }} " \Delta \varphi_{\text {atmo }}+\Delta \varphi_{\text {noise }}$. Knowing this, we can conclude that sum of atmospheric and noise phase effects is negligible compared to what is caused by displacement from a powerful underground explosion h-bomb. Therefore, the phase associated with the displacement was calculated by subtracting the topographic phase from the phase difference:

$$
\Delta \varphi_{\text {defo }}=\Delta \varphi-\Delta \varphi_{\text {topo }}
$$

Subtraction of the topographic phase was used to calculate the phase associated with displacement in radar image processing. The topographic phase depends on the perpendicular baseline $B_{\perp}$ [16] (See Fig. 3). The topographic phase is calculated as follows:

$$
\Delta \varphi_{\text {topo }}=\frac{4 \pi}{\lambda} \cdot \frac{B_{\perp}}{R \sin (\theta)} h
$$

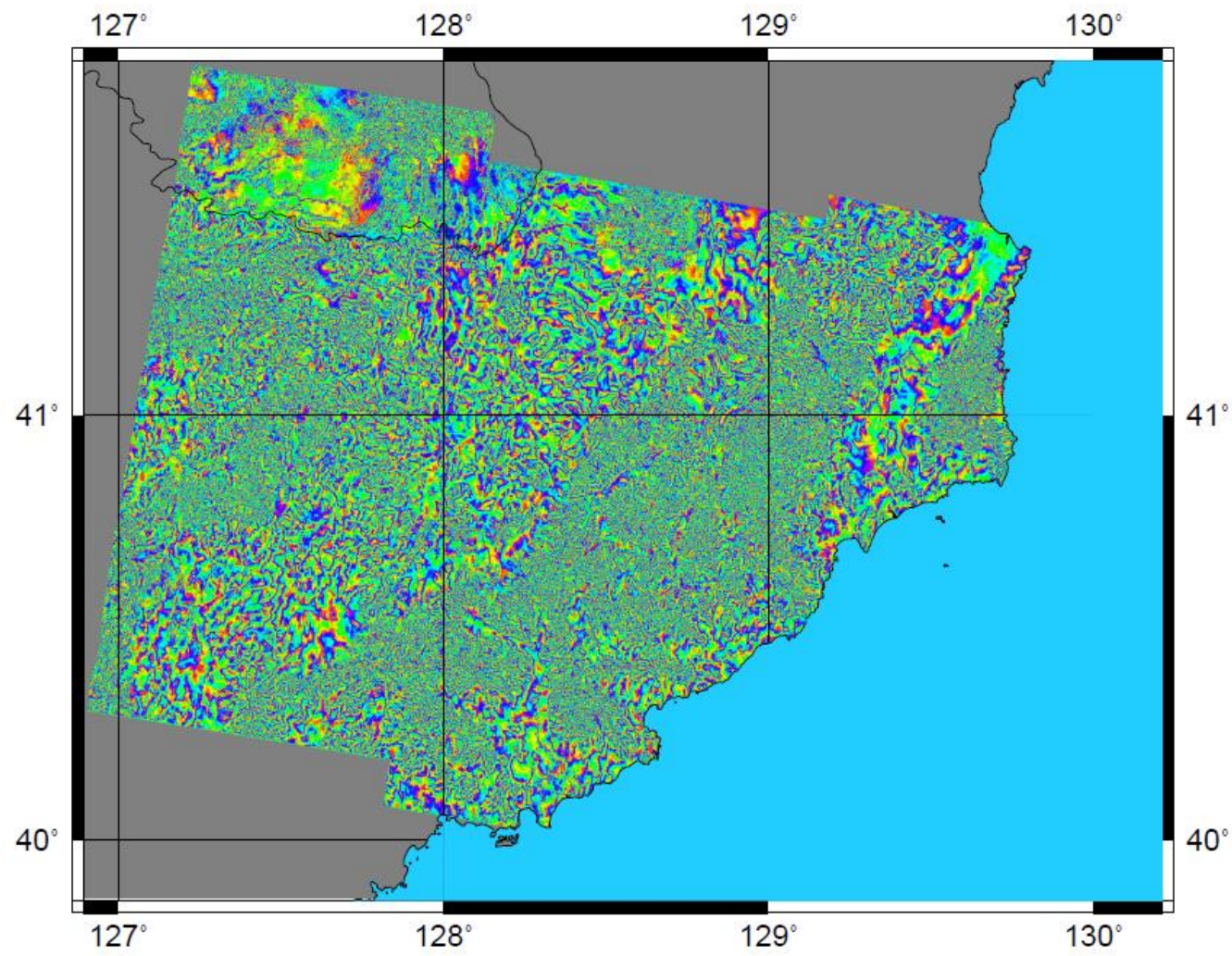

Figure 2. Geocoded image of the phase difference with the subtraction of the topographic component obtained from Sentinel-1B data with a descending orbit for Hamgyong province (August 18 and

September 10, 2017)

The procedure for subtracting the topographic phase was performed using a digital elevation model (DEM) and phase difference image. The DEM image contains the height value $h$. The DEM image is used to calculate the topographic phase component $\Delta \varphi_{\text {topo }}$ [17-19].

The phase associated with displacement can be distorted by noise. Noise can occur due to temporal decorrelation, geometric decorrelation, volume scattering, and processing error. The Goldstein filter was used to eliminate or partially reduce the phase noise effect [20-24]. 


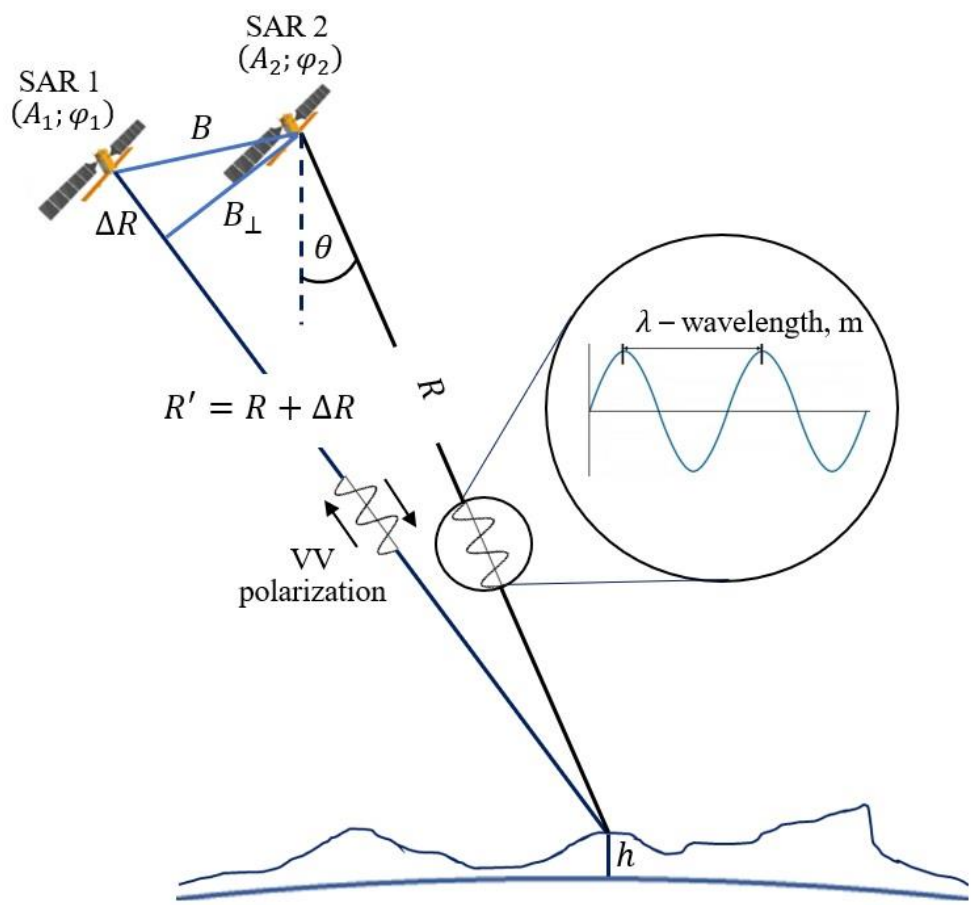

Figure 3. Application of Sentinel-1A/B remote sensing radar satellites for obtaining phase difference images from before (SAR 1) and after (SAR 2) underground explosion h-bomb

To generate an interferogram in the GTMSAR package, we need to run the script "p2p_S1_TOPS_Frame.csh", the command line looks like this for the first pair of the VV polarization interferogram for August 17 and September 10, 2020.

p2p S1 TOPS Frame.csh

S1B_IW_SLC_1SDV_20170817T213025_20170817T213052_006985_00C4D8_AF51.SAFE

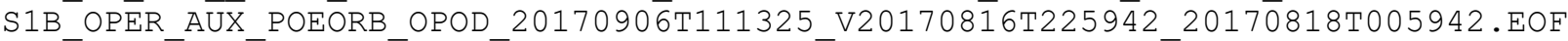

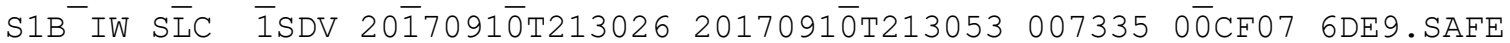

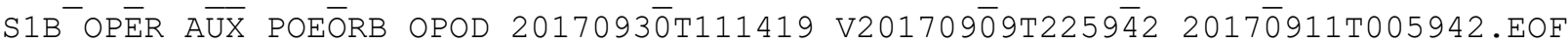

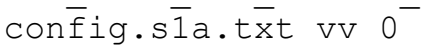

Before running the script, two folders called "raw" and "topo" should be prepared. The raw folder must contain Sentinel-1 satellite data in the directory with the name*. SAFE at the end. The topo folder must contain a DEM file generated from https://topex.ucsd.edu/gmtsar/demgen/.

The execution time of the "p2p_s1_tops_frame.csh" script depends on the computer's performance and takes the longest time, unlike other scripts. In general, the duration is 2 hours in VirtualBox Ubuntu 16.04 on an Intel ${ }^{\circledR}$ Core ${ }^{\mathrm{TM}} \mathrm{i} 7 \mathrm{-}-8565 \mathrm{U} 1.8 \mathrm{GHz}$ processor with RAM $8 \mathrm{~GB}$.

As a result of executing the script "p2p_s1_tops_frame. csh", three folders F1, F2, and F3, are created for each sub-swath IW1, IW2, and IW3. There are corresponding interferograms in those directories. In addition to these directories, the "merge" directory was formed, where the result of gluing interferograms of all sub-swaths is located (See Fig. 2).

The epicenter of an underground explosion h-bomb can be located in one or between sub-swaths. The epicenter underground explosion h-bomb cannot be visible on the filtered phase difference image, as shown in Figure 2. However, the epicenter location can be visible on absolute unwrapped phase difference and line-of-sight displacement images (See Fig. 4-5).

Using the script "snaphu_interp.csh" in the GMTSAR package, the phase difference image is converted to an absolute unwrapped phase, the program code was developed by Chen and Zebker [25-27]. This script is written in $\mathrm{C}$ and must be executed separately. Radar image processing using script "snaphu_interp.csh" demands a long period and required much memory. To decrease processing time, we need to decrease the area for processing by selecting the area in range-azimuth coordinates "0/25000/0/13072", where, the range from 0 to 25000 azimuth from 0 to 13072 . 
The absolute unwrapped phase difference image has a negative part - 6 and a positive part up to 44 radians in Figure 4. The script "0.10" value means that the part of the image with coherence less than 0.1 will be masked and will not be subjected to absolute unwrapped phase difference calculation.

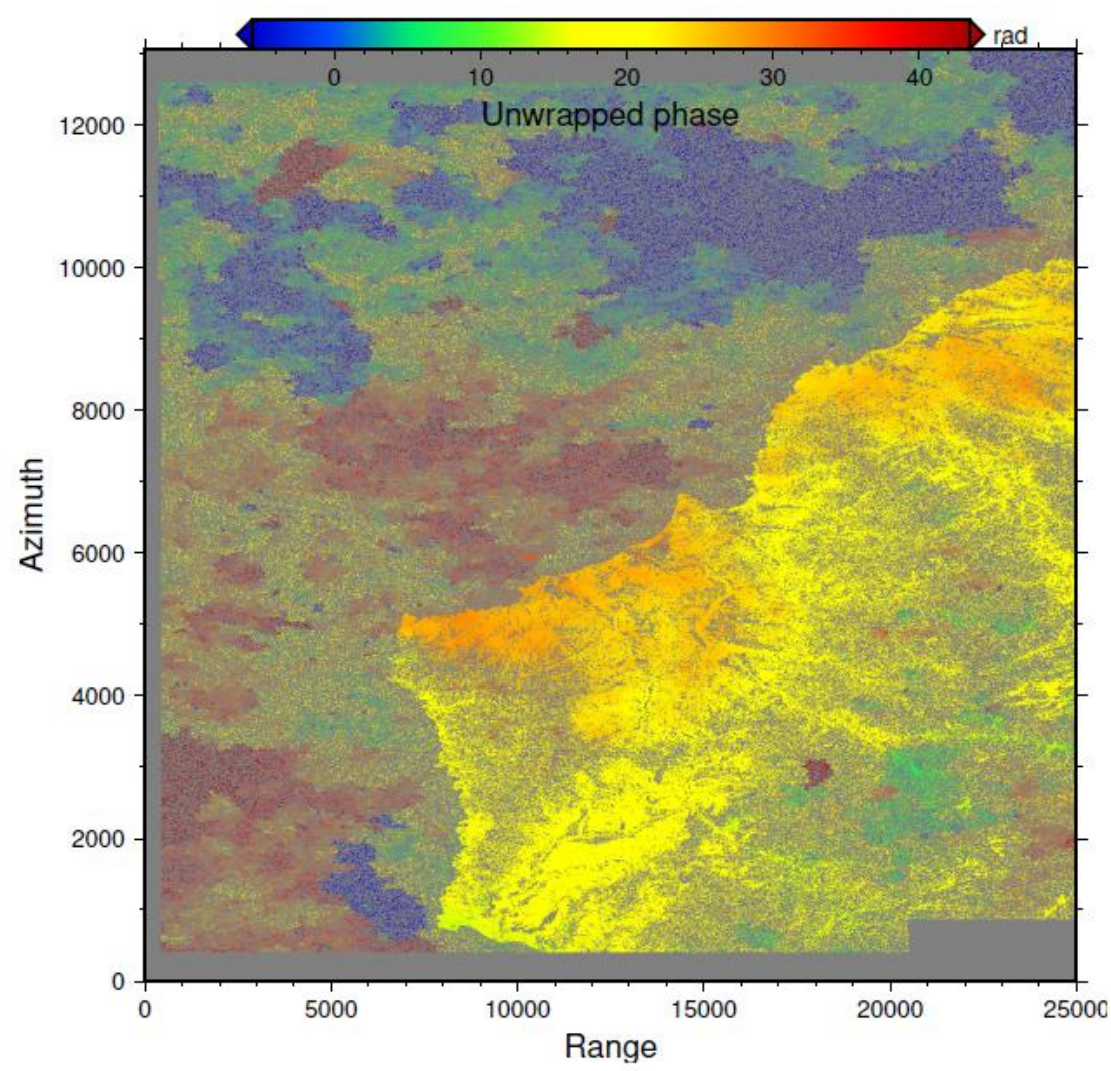

Figure 4. The absolute unwrapped phase difference of IW1-IW2 sub-swath Sentinel-1B with a descending orbit for Hamgyong province, North Korea (August 17 and September 10, 2017)

The following script is "geocode. csh .10" calculates the line-of-sight displacement image (See Fig. 5) and georeferences all data from radar coordinates (range, azimuth) to latitude and longitude geographic coordinates, along with kml-files for Google Earth.

geocode.csh .10

The line-of-sight displacement $\boldsymbol{d}_{\text {los }}$ radar is the difference between the distances before and after an underground explosion h-bomb, the shortest way a radio wave propagates from the antenna to the Earth's surface and back. Displacement on line-of-sight may differ depending on ascending (asc) and descending (desc) orbits of Sentinel-1A/B satellite $\boldsymbol{d}_{\text {los }}^{\text {asc }}, \boldsymbol{d}_{\text {los }}^{\text {desc }}$. These displacements at each point of the Earth's surface depend on the radio waves angle of incidence for the ascending and descending orbits $\boldsymbol{\theta}_{\text {asc }}, \boldsymbol{\theta}_{\text {desc }}$, respectively. The essence of line-of-sight displacement caused by the land subsidence is illustrated in Figure 6 when deforming down $\boldsymbol{d}^{\text {down }}$. 


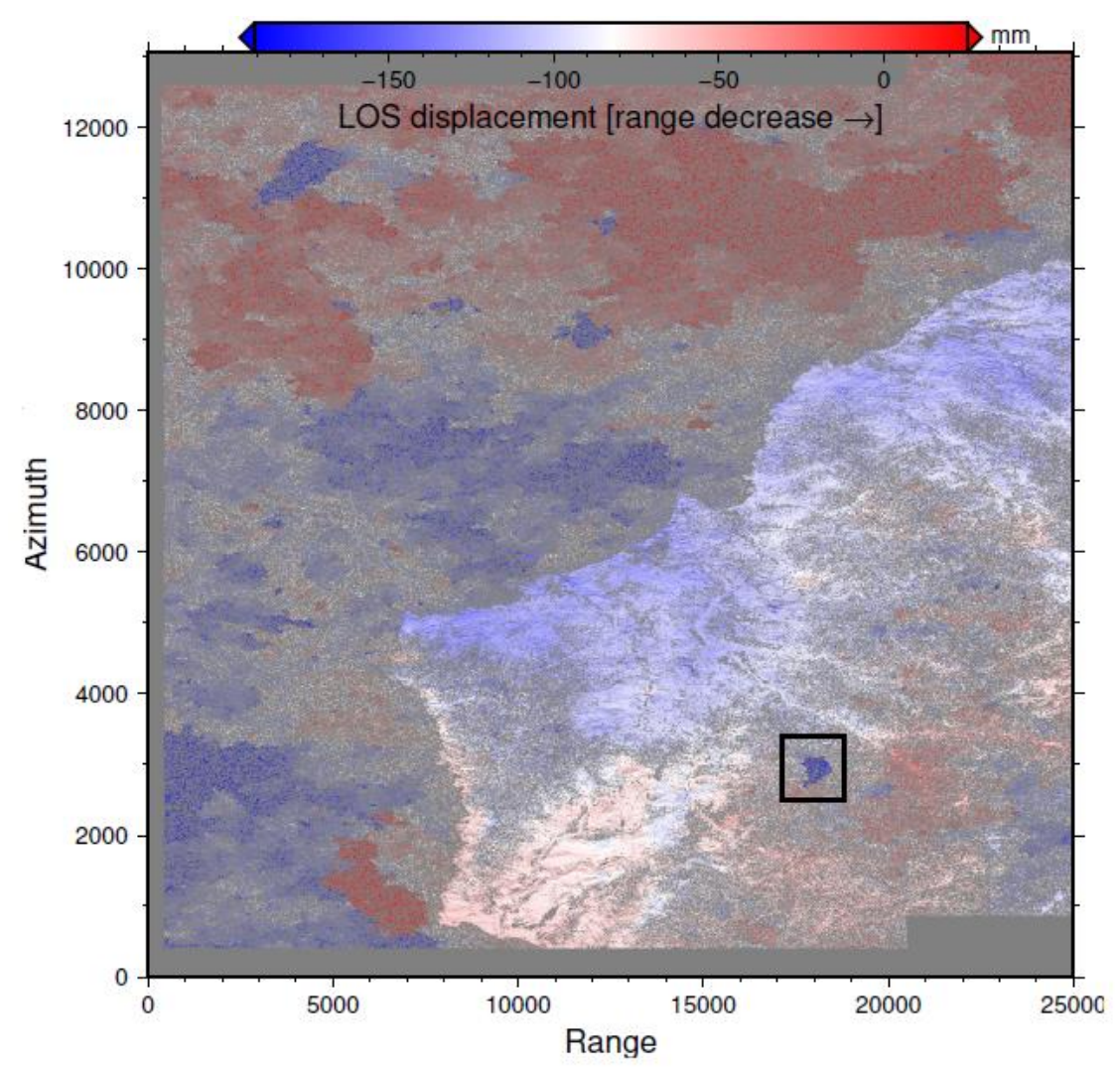

Figure 5. The line-of-sight displacement image of IW1-IW2 sub-swath Sentinel-1B with a descending orbit for Hamgyong province, North Korea (August 17 and September 10, 2017). Black frame: Location of the epicenter underground explosion h-bomb.

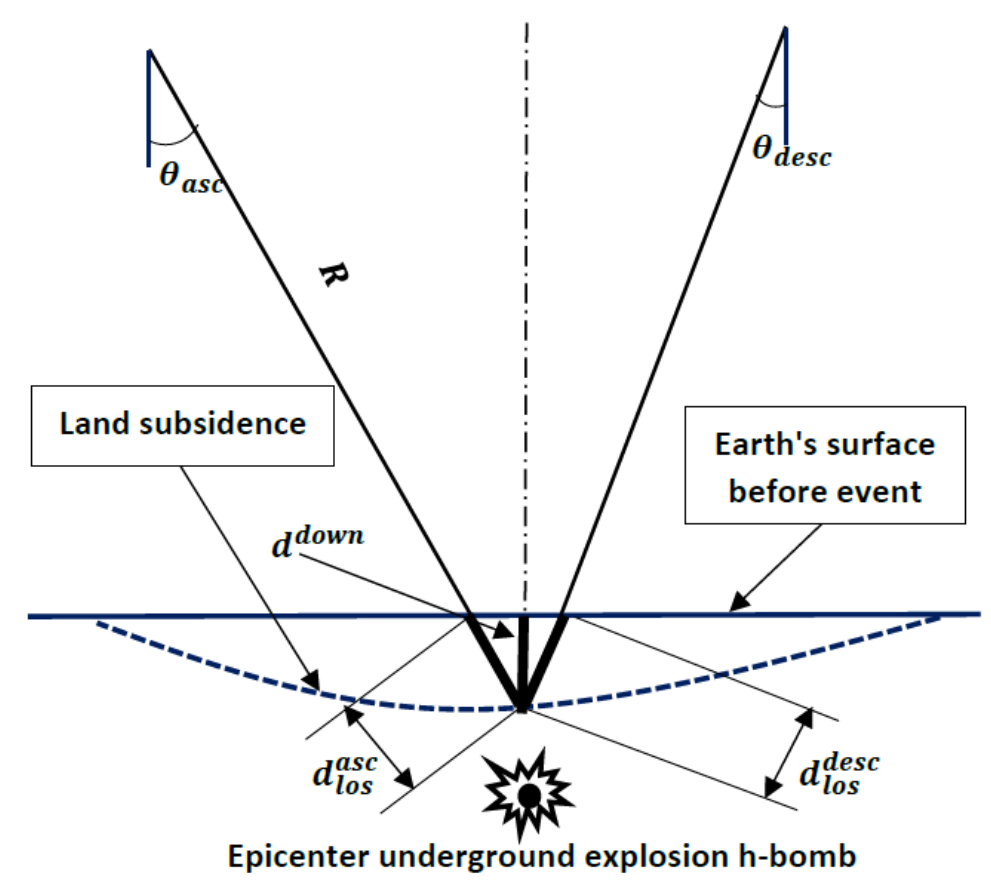

Figure 6. An image illustrating the land subsidence caused by underground explosion h-bomb

Thus, the radar image processing algorithm consists of the following operations shown in the block diagram in Figure 7. 


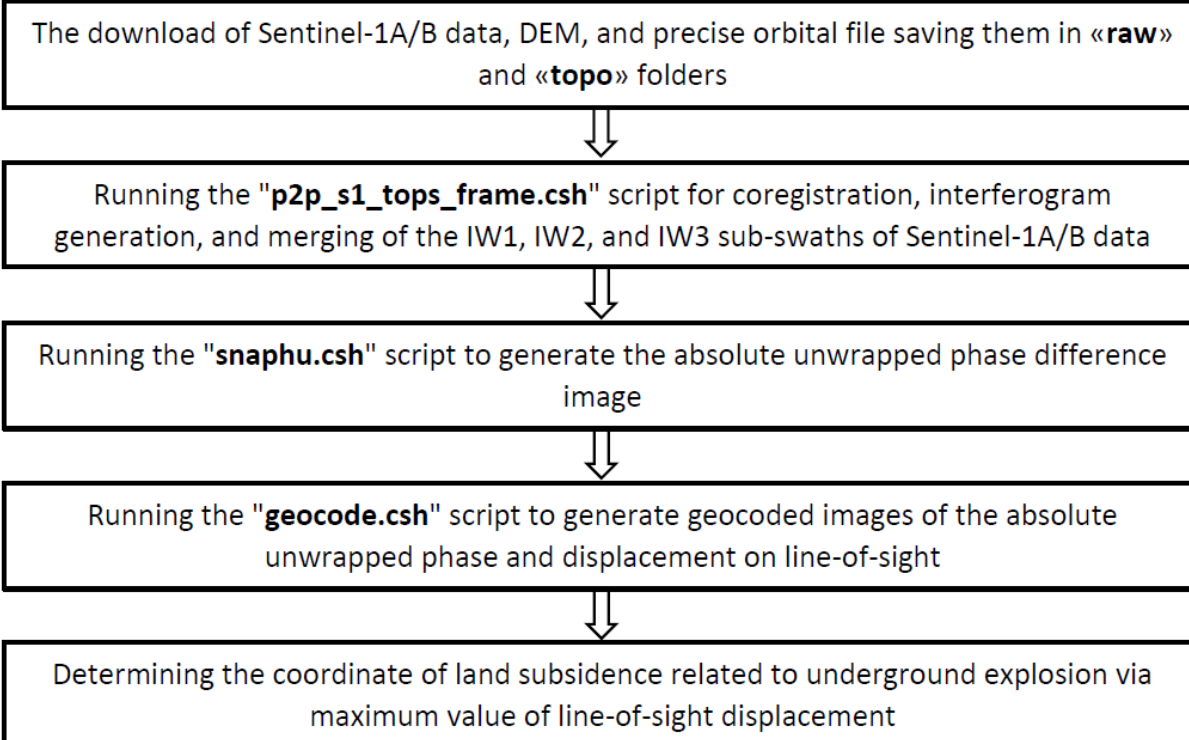

Figure 7. Radar image processing algorithm for determining the epicenter of an underground explosion hbomb coordinates based on maximum line-of-sight displacement using Sentinel-1A/B satellite data

\section{Results}

Various line-of-sight displacement images of Sentinel-1B data with descending orbits were applied to determine the epicenter of the underground explosion h-bomb. The maximum displacement modulus determined the coordinates of the epicenter. The displacement values have both positive and negative values. We need to select one, which has the absolute maximum value. The results of determining the epicenter coordinate through the maximum line-of-sight displacement module for underground explosion h-bomb using Sentinel-1A from the ascending orbit are presented in Figures 8-9.
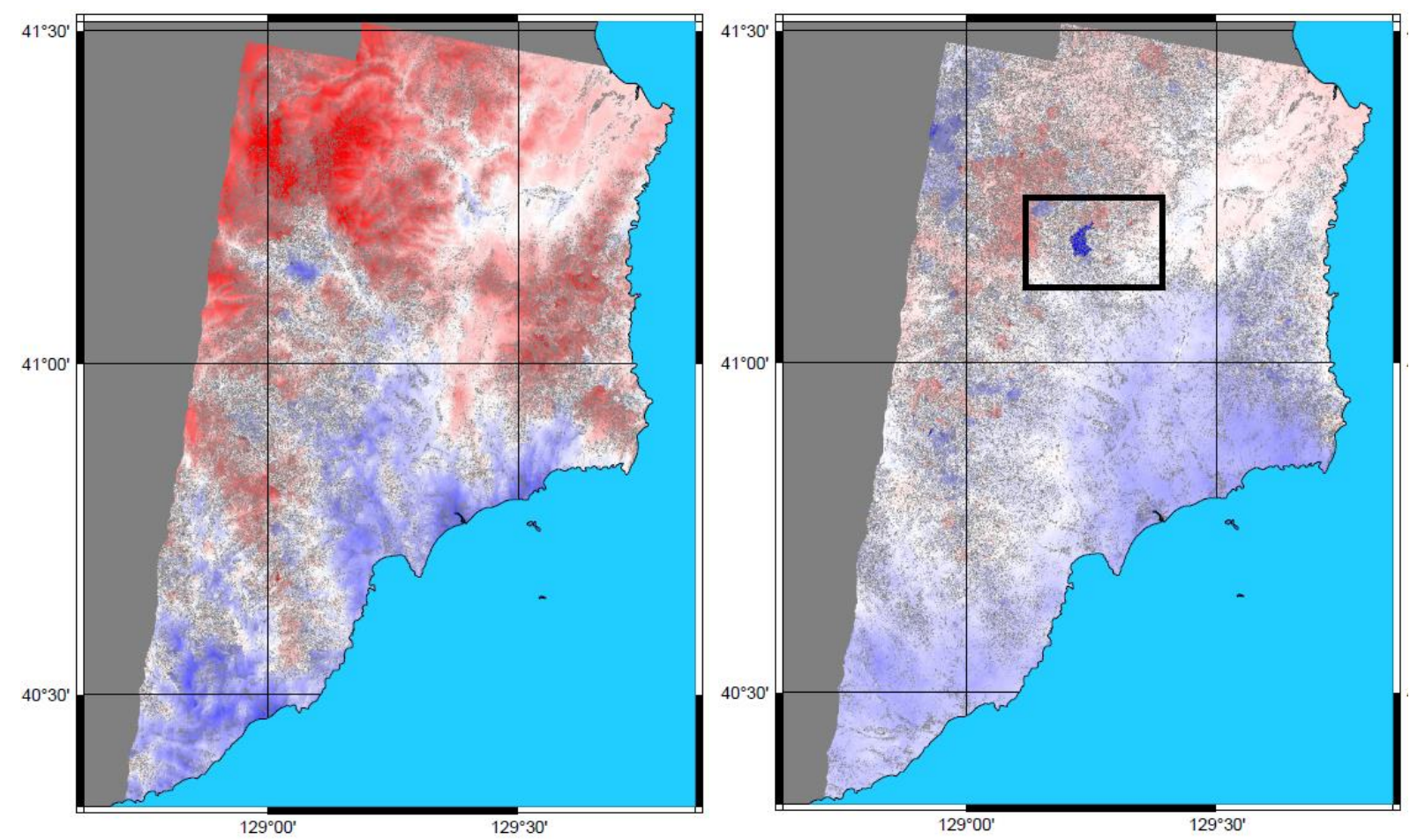

Figure 8. Geocoded line-of-sight displacement images of Sentinel-1B data with descending orbit. Left side: The first pair for dates, August 29 and September 10, 2017. Right part: The second pair for dates, August 17 and September 10, 2017. Black frame: Existence of epicenter or zone underground explosion h-bomb. 

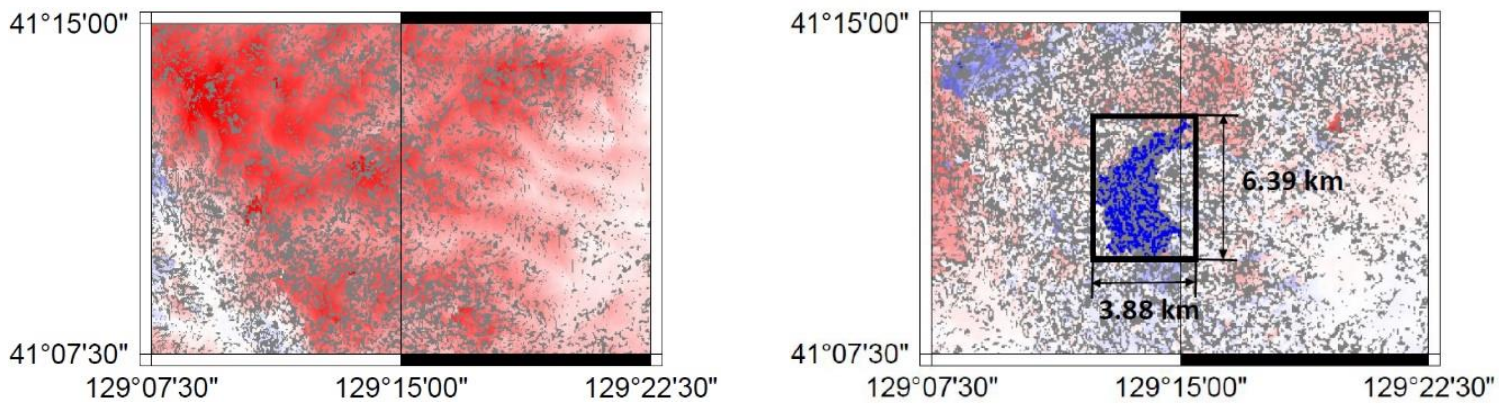

Figure 9. Zone underground explosion h-bomb from line-of-sight displacement image. Left side: The first pair for dates, August 29 and September 10, 2017. Right part: The second pair for dates, August 17 and September 10, 2017.

According to the Journal of Geophysical Research, the sixth nuclear test's power was about 250 kilotons of TNT equivalent in North Korea, 2017 [3]. Such an explosion could produce significant changes in the relief of the Earth's surface, which would be caused the maximum displacement related to land subsidence, as shown in Figure 8-9 (right part for pair, August 17 and September 10, 2017). Changes in displacement values from negative to positive were considered for the nuclear test. Negative values were used to determine the maximum changes. The absolute value displacement along the direct line-of-sight, which has negative values for the nuclear test, is $190 \mathrm{~mm}$. Significant displacement is visible in the right part of Figure 9; however, it is invisible in the left part of Figure 8 due to decorrelation and small perpendicular baseline (See Table 3 and Fig. 10). According to the line-of-sight displacement image, we can estimate the affected area, its dimensions: width $-3.88 \mathrm{~km}$ and length $-6.39 \mathrm{~km}$ (see Fig. 9 right part).

Table 3. Baseline estimation

\begin{tabular}{|c|c|c|c|c|c|}
\hline № & Study area & Orbits & Date before earthquake & Date after earthquake & $\begin{array}{c}\text { Perpendicular } \\
\text { baseline } B_{\perp}, \text { meter }\end{array}$ \\
\hline 1 & $\begin{array}{c}\text { Hamgyong } \\
\text { province, North } \\
\text { Korea }\end{array}$ & descending & 29.08 .2017 & 10.09 .2017 & 31.33 \\
\cline { 3 - 5 } 2 & descending & 17.08 .2017 & 10.09 .2017 & 68.1 \\
\hline
\end{tabular}

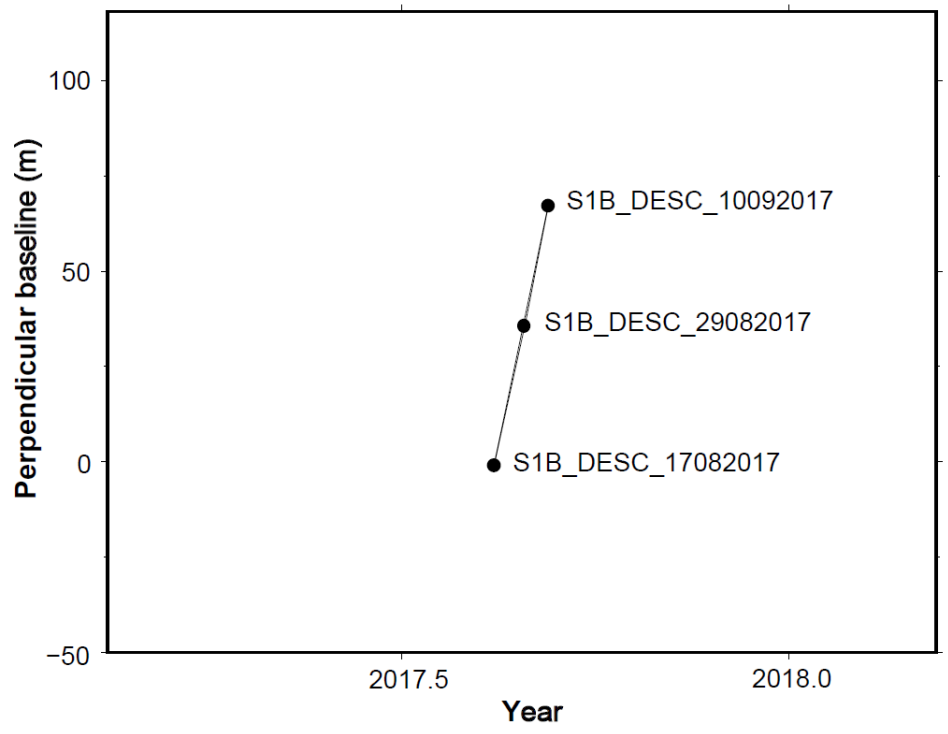

Figure 10. Perpendicular baseline relative to after the nuclear test event Sentinel-1B data on August 17, 2017 


\section{Conclusion}

Sentinel-1A/B radar remote sensing data is applicable for determining the nuclear test coordinate and affected area. At least one after and two before the event (the nuclear test date) scene Sentinel-1A/B data is needed to determine the epicenter and the affected area. Sentinel-1A/B data is more available than TerraSAR-X and ALOS-2 PALSAR radar data, which were applied for determining North Korea nuclear test epicenter and affected zone, previous research work.

We can find an underground nuclear test location via the maximum value (in millimeters) of line-ofsight displacement. This method allows us to determine the epicenter and the affected area using a line-ofsight displacement image. The high value associated with line-of-sight displacement was caused by the explosion of about 250 kilotons TNT equivalent. The underground nuclear test was like an earthquake with M6.3 magnitude.

Despite the GMTSAR package's availability, a drawback is the lack of a user-friendly interface in the ESA SNAP software. There are inconveniences, and they are associated with the manual launch of GMTSAR scripts and pre-preparation of data. This GMTSAR package is more intended for experts than ordinary users who do not have enough experience with the Linux terminal commands. Radar image processing in GMTSAR creates displacement images and determines the maximum displacement coordinates take less elapsed time than ESA SNAP. The GMTSAR package is undemanding to use more powerful computers than other Windows operation systems. A pair of Sentinel-1A/B data takes up 14 gigabytes of data, and it can increase several times due to data processing, so more capacious memory drives are needed. Therefore, more productive computing systems are required to work with such data arrays in some cases. We need supercomputer or cloud computing resources with more RAM and highspeed SSD-based hard drives for fast, automatic radar image processing, a program code that implements the entire algorithm as in Figure 7, and a friendly interface for interacting with radar remote sensing data.

\section{References}

[1] P. Gaebler et al., "A multi-technology analysis of the 2017 North Korean nuclear test," Solid Earth, vol. 10, no. 1, pp. 59-78, Jan 15 2019, doi: 10.5194/se-10-59-2019.

[2] D. Tian, J. Yao, and L. Wen, "Collapse and Earthquake Swarm After North Korea's 3 September 2017 Nuclear Test," Geophysical Research Letters, vol. 45, no. 9, pp. 3976-3983, May 16 2018, doi: 10.1029/2018gl077649.

[3] J. Liu, L. Li, J. Zahradnik, E. Sokos, C. Liu, and X. Tian, "North Korea's 2017 Test and its Nontectonic Aftershock," Geophysical Research Letters, vol. 45, no. 7, pp. 3017-3025, Apr 16 2018, doi: 10.1002/2018gl077095.

[4] T. Wang et al., "The rise, collapse, and compaction of Mt. Mantap from the 3 September 2017 North Korean nuclear test," Science, vol. 361, no. 6398, pp. 166-170, Jul 13 2018, doi: 10.1126/science.aar7230.

[5] S. C. Myers, S. R. Ford, R. J. Mellors, G. Ichinose, and S. Baker, "Absolute Locations of the North Korean Nuclear Tests Based on Differential Seismic Arrival Times and InSAR," Seismological Research Letters, vol. 89, no. 6, pp. 2049-2058, Nov-Dec 2018, doi: 10.1785/0220180123.

[6] K. M. Sreejith, R. Agrawal, and A. S. Rajawat, "Constraints on the location, depth and yield of the 2017 September 3 North Korean nuclear test from InSAR measurements and modelling," Geophysical Journal International, vol. 220, no. 1, pp. 345-351, Jan 2020, doi: 10.1093/gji/ggz451.

[7] W.-J. Lee, J. Sun, H.-S. Jung, S.-C. Park, D. K. Lee, and K.-Y. Oh, "Detection of Surface Changes by the 6th North Korea Nuclear Test Using High-resolution Satellite Imagery," Korean Journal of Remote Sensing, vol. 34, no. 6, pp. 1479-1488, Dec 2018, doi: 10.7780/kjrs.2018.34.6.4.2.

[8] W.-K. Baek, H.-S. Jung, and T. S. Kim, "Satellite radar observation of large surface collapses induced by the 2017 North Korea nuclear test," Scientific Reports, vol. 10, no. 1, Oct 20 2020, Art no. 17833, doi: 10.1038/s41598-020-74957-2.

[9] E. J. Fielding et al., "Surface Deformation Related to the $2019 \mathrm{M}-\mathrm{w} 7.1$ and 6.4 Ridgecrest Earthquakes in California from GPS, SAR Interferometry, and SAR Pixel Offsets," Seismological Research Letters, vol. 91, no. 4, pp. 2035-2046, Jul 2020, doi: 10.1785/0220190302. 
[10] Z. C. Huang, G. H. Zhang, X. J. Shan, W. Y. Gong, Y. F. Zhang, and Y. C. Li, "Co-Seismic Deformation and Fault Slip Model of the 2017 Mw 7.3 Darbandikhan, Iran-Iraq Earthquake Inferred from DInSAR Measurements," Remote Sensing, vol. 11, no. 21, Nov 2019, Art no. 2521, doi: $10.3390 /$ rs11212521.

[11] S. Plank, "Rapid Damage Assessment by Means of Multi-Temporal SAR - A Comprehensive Review and Outlook to Sentinel-1," Remote Sensing, vol. 6, no. 6, pp. 4870-4906, Jun 2014, doi: $10.3390 /$ rs6064870.

[12] S. Wang, Y. Zhang, Y. Wang, J. Jiao, Z. Ji, and M. Han, "Post-seismic deformation mechanism of the July 2015MW 6.5 Pishan earthquake revealed by Sentinel-1A InSAR observation," Scientific reports, vol. 10, no. 1, pp. 18536-18536, 2020 Oct 2020, doi: 10.1038/s41598-020-75278-0.

[13] M. LazeckY et al., "Displacements Monitoring over Czechia by IT4S1 System for Automatised Interferometric Measurements Using Sentinel-1 Data," Remote Sensing, vol. 12, no. 18, Sep 2020, Art no. 2960, doi: 10.3390/rs12182960.

[14] T. Gatsios, F. Cigna, D. Tapete, V. Sakkas, K. Pavlou, and I. Parcharidis, "Copernicus Sentinel-1 MTInSAR, GNSS and Seismic Monitoring of Deformation Patterns and Trends at the Methana Volcano, Greece," Applied Sciences-Basel, vol. 10, no. 18, Sep 2020, Art no. 6445, doi: 10.3390/app10186445.

[15] X. H. Xu, D. T. Sandwell, and B. Smith-Konter, "Coseismic Displacements and Surface Fractures from Sentinel-1 InSAR: 2019 Ridgecrest Earthquakes," Seismological Research Letters, vol. 91, no. 4, pp. 1979-1985, Jul 2020, doi: 10.1785/0220190275.

[16] D. Suresh and K. Yarrakula, "InSAR based deformation mapping of earthquake using Sentinel 1A imagery," Geocarto International, vol. 35, no. 5, pp. 559-568, Apr 2020, doi: 10.1080/10106049.2018.1544289.

[17] H. Cui and X. J. Zha, "Parallel Image Registration Implementations for GMTSAR Package," Seismological Research Letters, vol. 89, no. 3, pp. 1129-1136, May-Jun 2018, doi: 10.1785/0220170171.

[18] B. Zhang, J. Y. Li, and H. R. Ren, "Using Phase Unwrapping Methods to Apply D-InSAR in Mining Areas," Canadian Journal of Remote Sensing, vol. 45, no. 2, pp. 225-233, Mar 2019, doi: 10.1080/07038992.2019.1583097.

[19] J. M. D. Blasco, M. Foumelis, C. Stewart, and A. Hooper, "Measuring Urban Subsidence in the Rome Metropolitan Area (Italy) with Sentinel-1 SNAP-StaMPS Persistent Scatterer Interferometry," Remote Sensing, vol. 11, no. 2, Jan 2019, Art no. 129, doi: 10.3390/rs11020129.

[20] C. Danisor and A. Pepe, "Comparative study of SAR interferometric phase filtering algorithms," in Advanced Topics in Optoelectronics, Microelectronics, and Nanotechnologies Ix, vol. 10977, M. Vladescu, R. Tamas, and I. Cristea Eds., (Proceedings of SPIE, 2018.

[21] A. Tripathi and S. Kumar, Effect of Phase Filtering on Interferometry based Displacement Analysis of Cultural Heritage Sites (2018 5th leee Uttar Pradesh Section International Conference on Electrical, Electronics and Computer Engineering). 2018, pp. 18-22.

[22] Q. Q. Feng, H. P. Xu, Z. F. Wu, Y. A. You, W. Liu, and S. Q. Ge, "Improved Goldstein Interferogram Filter Based on Local Fringe Frequency Estimation," Sensors, vol. 16, no. 11, Nov 2016, Art no. 1976, doi: 10.3390/s16111976.

[23] A. Mestre-Quereda, J. M. Lopez-Sanchez, J. Selva, and P. J. Gonzalez, "An Improved Phase Filter for Differential SAR Interferometry Based on an Iterative Method," leee Transactions on Geoscience and Remote Sensing, vol. 56, no. 8, pp. 4477-4491, Aug 2018, doi: 10.1109/tgrs.2018.2820725.

[24] Z. Y. Suo, J. Q. Zhang, M. Li, Q. J. Zhang, and C. Fang, "Improved InSAR Phase Noise Filter in Frequency Domain," leee Transactions on Geoscience and Remote Sensing, vol. 54, no. 2, pp. 1185-1195, Feb 2016, doi: 10.1109/tgrs.2015.2476355.

[25] C. W. Chen and H. A. Zebker, "Network approaches to two-dimensional phase unwrapping: intractability and two new algorithms," Journal of the Optical Society of America a-Optics Image Science and Vision, vol. 17, no. 3, pp. 401-414, Mar 2000, doi: 10.1364/josaa.17.000401.

[26] C. W. Chen and H. A. Zebker, "Phase unwrapping for large SAR interferograms: Statistical segmentation and generalized network models," leee Transactions on Geoscience and Remote Sensing, vol. 40, no. 8, pp. 1709-1719, Aug 2002, doi: 10.1109/tgrs.2002.802453. 
[27] C. W. Chen and H. A. Zebker, "Two-dimensional phase unwrapping with use of statistical models for cost functions in nonlinear optimization," Journal of the Optical Society of America a-Optics Image Science and Vision, vol. 18, no. 2, pp. 338-351, Feb 2001, doi: 10.1364/josaa.18.000338. 